\title{
Análisis socioespacial de los Institutos Tecnológicos federales de la Ciudad de México
} Socio-spatial Analysis of the Federal Technological Institutes in Mexico City

\author{
Diego Ángeles Colín \\ Institute of Education, University College London, Reino Unido \\ dangeles9@gmail.com \\ Gustavo Mejía Pérez \\ Universidad Pedagógica Nacional, México \\ cabezahidra@gmail.com
}

\section{RESUMEN}

Entre 2008 y 2012 se establecieron doce Institutos Tecnológicos Federales en la Ciudad de México para ampliar las oportunidades educativas en las alcaldías con mayor rezago en educación superior. En el presente artículo exploramos la pertinencia socioespacial de este subsistema educativo y buscamos arrojar luz sobre dos problemáticas actuales del mismo. Nuestro análisis se basa en el uso de un sistema de información geográfico (Q-GIS) que incorpora las siguientes variables a nivel de alcaldías y municipios: número de instituciones de educación superior, Índice de Desarrollo Humano, y número de jóvenes de entre 15 y 24 años por municipio o alcaldía. El análisis descriptivo muestra que los IT en la Ciudad de México tienden a ubicarse en las alcaldías con menores niveles de desarrollo regional y mayor número de jóvenes.

Palabras clave: educación superior tecnológica, geografía de la educación, descentralización educativa

\begin{abstract}
Between 2008 and 2012 twelve technological institute were established in Mexico City to widen the educational opportunities in boroughs with low educational attainment. In this article we explore the socio-spatial pertinence of this educational subsystem and shed light on two common problems within the institutes. Our analysis is based on a geographic information system (Q-GIS) that includes the following variables at the municipal level: number of higher education institutions, Human Development Index and amount of young population ages 15 and 24 . The descriptive analysis shows that the IT are located in boroughs with the lowest IDH and high concentration of young people.
\end{abstract}

Keywords: technological higher education, geography of education, educational decentralization 


\section{INTRODUCCIÓN}

En 2008, el gobierno federal inauguró los tres primeros planteles del sistema de institutos tecnológicos federales (IT) en la Ciudad de México. En los tres años siguientes se establecieron nueve institutos tecnológicos más, sumando en total doce planteles en la capital mexicana. Esto aumentó la oferta en educación superior tecnológica en la ciudad que hasta ese entonces estaba representada sólo por el Instituto Politécnico Nacional (IPN). Su establecimiento en la ciudad respondía a las necesidades de ampliar las oportunidades educativas para el creciente número de estudiantes que no encontraban espacios en las universidades públicas de mayor demanda, como la Universidad Nacional Autónoma de México (UNAM) o el mismo IPN, así como de atender la demanda local de las alcaldías de la ciudad con mayor rezago en educación superior (ES) (DGEST, 2009). Sin embargo, a la fecha los doce planteles funcionan por debajo de su capacidad, al mismo tiempo que se observa un crecimiento desigual en la matrícula de cada institución.

En el presente artículo exploramos la pertinencia socioespacial de los institutos tecnológicos en la Ciudad de México y buscamos arrojar luz sobre su baja demanda mediante visualizaciones de datos georreferenciados (Hillman, 2017). Usamos las siguientes variables, todas a nivel municipal: número de instituciones de educación superior, Índice de Desarrollo Humano, y número de jóvenes de entre 15 y 24 ańos. Al mismo tiempo, buscamos contribuir al conocimiento de la situación actual y problemáticas de la educación superior tecnológica (EST) en la capital mexicana.

\section{LA IMPORTANCIA DE LA GEOGRAFÍA EN EL ACCESO A LA EDUCACIÓN SUPERIOR}

La Ciudad de México es un espacio privilegiado en cuanto a infraestructura y oferta en educación superior. Es sede de las universidades públicas más grandes y de mayor tradición en el país como la UNAM y el IPN, así como de un gran número de universidades privadas, escuelas de posgrado y centros de investigación. Adicionalmente, la ciudad concentra la oferta del sistema de educación superior en línea 
más grande del país, la Universidad Abierta y a Distancia de México (UAyDM). Con esta vasta oferta, la matrícula en ES en la Ciudad de México y su zona metropolitana supera la media nacional (De Garay, 2003), al tiempo que se estima que la oferta de lugares en las IES es mayor que la demanda a nivel regional (Malo y Hernández, 2014). Sin embargo, la desigualdad de acceso a la ES persiste en la ciudad. Por ejemplo, mientras que en alcaldías como Benito Juárez y Miguel Hidalgo la proporción de la población con estudios superiores supera 50\%, en Tláhuac y Milpa Alta, al suroriente de la ciudad, la cifra es de alrededor de 20 y 13\%, respectivamente (INEGI, 2015b). Una posible explicación a esta desigualdad educativa podría radicar en las características geográficas de la oferta de instituciones de educación superior (IES) en la ciudad.

La geografía es un factor clave de las oportunidades de acceso a la educación superior, en especial para los jóvenes más desfavorecidos. Por ejemplo, en Estados Unidos y Australia se ha sugerido que las probabilidades de acceder a la educación terciaria aumentan con la presencia de una o más IES en la localidad de residencia (Hillman, 2016). Se han propuesto dos explicaciones a esta asociación; ya sea que los establecimientos académicos realicen frecuentemente actividades de promoción en la comunidad circundante que estimulen las aspiraciones de los jóvenes, o que la cercanía entre una universidad y el hogar ayude a reducir los costos tanto económicos (los relativos al traslado) como emocionales (distancia de la familia y la comunidad) que enfrentan los estudiantes de menores recursos (Lopez-Turley, 2009).

Además, se ha sugerido que las oportunidades educativas están asociadas a los patrones espaciales de estratificación social, étnica y de clase, lo que en la mayoría de las veces resulta en limitaciones estructurales de elección para las poblaciones más desfavorecidas. Por ejemplo, en Estados Unidos cerca de 57\% de los alumnos universitarios de nuevo ingreso se inscriben en una institución localizada en un radio no mayor a los $80 \mathrm{~km}$ de distancia de su hogar (Hillman y Weichman, 2016). Sin embargo, Hillman (2016) encuentra que grupos minoritarios como los latinos suelen elegir IES de corte técnico-vocacional de dos años (community colleges) debido a que son las que se encuentran más cercanas a sus domicilios. Así, la ubi- 
cación espacial contribuye a la construcción de sus oportunidades educativas y de vida. Esta desigualdad de oportunidades de acceso se hace aún más patente cuando el número de IES en una comunidad es reducido o nulo.

A lo largo del siglo XX, la oferta en ES de la Ciudad de México experimentó un continuo proceso de distribución territorial, dejando atrás el modelo de barrio universitario para pasar a un modelo policéntrico (Terrazas y Rodríguez, 2017). Diversos estudios han observado la tendencia de las IES tanto públicas como privadas a concentrarse en la zona centro-sur de la ciudad, específicamente en las alcaldías Benito Juárez, Coyoacán, Cuauhtémoc y Miguel Hidalgo (Terrazas y Rodríguez, 2017, Taborga, 2002). A pesar de la falta de mayor evidencia del efecto negativo que esta concentración de la oferta pueda tener sobre las oportunidades de acceso a la ES entre los habitantes de la ciudad, en los últimos años las políticas públicas en ES locales se han orientado a descentralizar la oferta educativa. De esta forma, las autoridades en la ciudad (federales y locales) suelen elegir demarcaciones territoriales con baja o nula oferta educativa como sedes de los nuevos establecimientos académicos. Un ejemplo de esta política de descentralización, lo que aquí denominamos "equidad espacial", son los doce IT de la ciudad

\section{Las políticas de descentralización educativa y los institutos tecnológicos}

En el país, la oferta en educación superior está altamente concentrada. De acuerdo con datos oficiales, únicamente $16 \%$ de los 2458 municipios del país cuentan con algún tipo de oferta de estudios superiores (Conaculta, 2012). Las oportunidades de acceso a la ES tienden a ser mucho mayores en zonas urbanas y metropolitanas en comparación con zonas rurales y de baja densidad poblacional (Ortega y Casillas, 2013, Gil Antón et al., 2009). Este dominio educativo de las urbes sobre otros territorios se puede entender como resultado histórico del sistema de organización dominante en el país, el centralismo. Así, a lo largo del siglo XX, la oferta y demanda de estudios superiores tendieron a concentrarse en las principales ciudades de México, debido a que en ellas se aglomeraron la in- 
fraestructura educativa, así como la industria y la burocracia (Mier y Rabell, 2003, Taborga, 2003). Dado este panorama, las políticas de expansión de la matrícula en ES han estado estrechamente vinculadas a la democratización territorial del sistema educativo, proceso coordinado desde el gobierno y las instituciones académicas mediante las políticas de descentralización educativas (Didou, 2011).

Las primeras estrategias de descentralización territorial de las IES surgieron al margen de la masificación del sistema de ES en los años setenta e inicios de los ochenta (Didou, 1997). En una primera etapa, las políticas de distribución de las IES se diseñaron a partir de un enfoque regional y estatal. Las universidades públicas estatales comenzaron su expansión al interior de sus estados de jurisdicción con la creación de unidades satélite en diferentes municipios. Más tarde, en los ańos noventa, se establecieron nuevos sistemas con modalidades de gestión y financiamiento de base estatal, como los institutos tecnológicos descentralizados o los colegios de estudios científicos y tecnológicos estatales, Cecytes (Weiss y Bernal, 2013, Rodríguez, 1998). Posteriormente, a inicios del siglo XXI, predominó el enfoque municipal-comunitario de la distribución con el surgimiento de las universidades interculturales y las universidades politécnicas, al mismo tiempo que la educación superior a distancia surgió como una alternativa viable frente a los desafíos territoriales y económicos de la expansión de la infraestructura educativa.

Estas políticas de descentralización han reducido las desigualdades territoriales de acceso a la ES. Como ejemplo de ello, entre 1990 y 2007 el número de municipios con al menos una IES pasó de 157 a 309 (Didou, 2011). No obstante, a pesar de este avance positivo en materia de equidad espacial, existen importantes limitaciones. Por un lado, se ha señalado que la descentralización de la oferta ha beneficiado principalmente a localidades semiurbanas y colindantes a zonas metropolitanas con bajos niveles de rezago social. Este es el caso de la expansión de las unidades desconcentradas de las universidades estatales en las últimas dos décadas (Mejía y Worthman, 2015). Al mismo tiempo, estas políticas se han encargado principalmente de distribuir la infraestructura educativa, dejando de lado los sesgos geográficos de la calidad de la oferta misma o de las trayectorias educativas y las condiciones socioeconómicas de los estudiantes 
(Didou, 2011). Como prueba de ello, se ha observado que algunas instituciones como las universidades tecnológicas o las unidades desconcentradas de las universidades autónomas no llegan a operar al máximo de su capacidad institucional (Mejía y Worthman, 2017, Gil Antón et al., 2009).

Los institutos tecnológicos públicos son parte fundamental de la educación superior tecnológica, oferta de nivel superior que se especializa primordialmente en el campo de las ingenierías y áreas relacionadas con la producción y gestión industrial. ${ }^{1}$ A nivel histórico, los institutos tecnológicos son un sistema pionero en la descentralización de la oferta educativa en México. Este subsistema nace en el norte de México, con el establecimiento de los primeros tecnológicos -entonces denominados regionales- en Durango y Chihuahua en 1948. Luego de varias décadas de crecimiento continuo, a inicios de los años noventa surgieron los institutos tecnológicos descentralizados, los cuales se caracterizan por tener mayor vínculo administrativo y de financiamiento con los gobiernos estatales, en comparación con los anteriores institutos que, desde sus inicios, ostentan exclusiva dependencia del ámbito federal.

Actualmente, el sistema de institutos tecnológicos tiene presencia en cada estado de México y en municipios no conurbados. En algunos municipios, los institutos tecnológicos llegan a representar la única opción disponible de ES (Didou, 1997). La clave de este logro está en el continuo interés y apoyo provenientes del gobierno federal desde los noventa, en el contexto de la restructuración de la industria mexicana, tras la firma del Tratado de Libre Comercio de América del Norte. A lo largo de dos décadas, el ritmo de crecimiento del sistema se aceleró gracias al aumento de su financiamiento, pasando de 100 planteles en 1991 a 256 en 2014. Al mismo tiempo, su estructura organizacional se ha fortalecido a lo largo de estos mismos ańos, primero con la mayor participación de los gobiernos estatales en la administración y financiamiento del sistema -a través de los institutos tecnológicos descentralizados- $\mathrm{y}$, segundo, al alcanzar un nivel más amplio de autonomía respecto de la adminis-

\footnotetext{
${ }^{1}$ Además de los institutos tecnológicos, en México la educación superior tecnológica incluye al Instituto Politécnico Nacional (IPN), a las Universidades Politécnicas y a las Universidades Tecnológicas (ver Ruiz-Larraguivel, 2011).
} 
tración federal con la creación del Tecnológico Nacional de México (TecNM), en 2014.

El TecNM coordina el financiamiento y administración de los 254 institutos del país, tanto descentralizados como federales, más seis centros de investigación. Es un órgano desconcentrado de la SEP que goza de autonomía técnica, académica y de gestión. Por el número de planteles y estudiantes inscritos, el TecNM es una de las instituciones de educación superior más grandes de Latinoamérica. Dentro de la EST, el TecNM destaca por su oferta de programas académicos de cuatro ańos y modalidades mixtas, mientras que otras opciones de EST ofrecen programas cortos de dos o tres ańos.

Heredero de los esfuerzos de descentralización territorial del sistema de institutos tecnológicos, el TecNM ha asumido como misión: "Ofrecer la más amplia cobertura educativa que asegure la igualdad de oportunidades para estudiantes en localidades aisladas y zonas urbanas marginadas, impulse la equidad, la perspectiva de género, la inclusión y la diversidad" (DOF, 2014).

En septiembre de 2008, el gobierno federal inauguró los tres primeros institutos tecnológicos de la Ciudad de México. A estos siguieron nueve planteles más, sumando un total de doce IT en la actualidad. El establecimiento del sistema de institutos tecnológicos en la capital del país tenía como objetivo principal ampliar las oportunidades educativas para el creciente número de estudiantes que no encontraban espacios en las universidades públicas de mayor demanda, como la UNAM o el IPN.

Aunado a la apertura de nuevos espacios educativos, los tecnológicos de la Ciudad de México buscaban reducir el rezago en educación superior de algunas alcaldías de la ciudad. Las alcaldías seleccionadas fueron Iztapalapa, Milpa Alta, Tláhuac, Álvaro Obregón y Gustavo A. Madero. Si bien algunas instituciones públicas como la Universidad Autónoma Metropolitana (UAM), fundada en 1974, o la Universidad Autónoma de la Ciudad de México (UACM) de 2001 habían nacido con la misión de descentralizar territorialmente la oferta educativa, con los tecnológicos se buscaba la atención directa a la demanda local de sus demarcaciones sedes. Esto último no limita a las instituciones a atender a estudiantes provenientes de otros municipios, aunque en algunos casos era una misión priorita- 
ria ante la falta de otras opciones en la zona o por el difícil acceso a la localidad.

En el contexto mexicano, algunos estudios han abordado la influencia de la geografía sobre las problemáticas educativas, en particular respecto de la distribución territorial de las instituciones (Terrazas y Rodriguez, 2017, Gil Antón et al., 2009) mientras que otros analizan factores como la movilidad espacial producida por y alrededor de la educación superior (Mejía, 2019). A pesar de estos avances, aquí sostenemos que la geografía dista aún de ser un ángulo de análisis de importancia dentro de la investigación educativa mexicana. Con ello en mente, nuestro trabajo busca contribuir al aumento del interés por la perspectiva geográfica en los estudios de la educación superior en el país.

En este sentido, el presente artículo explora la expansión de los IT en la Ciudad de México y la Zona Metropolitana del Valle de México (ZMVM) haciendo uso de algunas de las herramientas analíticas de la geografía de la educación (Hillman, 2017). Creemos que este subcampo ofrece herramientas originales desde las que se pueden elaborar marcos analíticos originales y enriquecedores sobre los fenómenos educativos. Al mismo tiempo, buscamos presentar una herramienta de trabajo que pueda ayudarnos a profundizar sobre el papel que juega el espacio en la distribución de oportunidades en el contexto mexicano. Nuestro objetivo primario es explorar la pertinencia socioespacial de las doce sedes en la Ciudad de México mediante un análisis descriptivo de su ubicación en relación con tres variables: número de instituciones de educación superior, Índice de Desarrollo Humano, y número de jóvenes de entre 15 y 24 años, todos por municipio o alcaldía. Por otra, buscamos arrojar luz sobre la baja demanda de los IT en la ciudad. En este trabajo tomamos como marco de referencia espacial a la ZMVM, por la vecindad de las entidades y la gran movilidad de estudiantes que existe en la región (Malo y Hernández, 2014).

\section{METODOLOGÍA}

Aquí sostenemos que la geografía de la educación debe entenderse como una invitación a capturar la dimensión espacial de los fenómenos educativos mediante diversos instrumentos de investigación 
y marcos de interpretación propios de la geografía humana. La metodología que aquí adoptamos tiene como fundamento el análisis geoespacial, una herramienta de exploración cuya principal premisa es la construcción de cartografías para la observación de diversas capas de datos cuantitativos (Hillman, 2017). Nos apoyamos en el software Quantum Geographic Information System o Q-GIS, un sistema de información geográfica (SIG) cuya principal función es analizar y visualizar datos georreferenciados de gran escala (Olaya, 2014). Sostenemos que las formas habituales de representar los datos en ciencias sociales, como los gráficos y las tablas, no permiten identificar todas las diversas formas en que el espacio influye sobre los procesos sociales y educativos. Por el contrario, los mapas obtenidos a través del SIG permiten visualizar, simultáneamente, propiedades espaciales tales como distancia, tendencias de distribución o concentración, procesos dinámicos de flujo y movilidad, y límites o radios de alcance de diversos fenómenos sociales y educativos.

\section{Capas de información}

Para este trabajo se recuperaron tres variables: la base de datos de las escuelas de ES del Sistema Nacional de Información de Escuelas (SNIE), ciclo escolar 2015-2016 (SEP) y que se transportó al software Q-GIS (Versión 3.0), el cual, a su vez, trabajó a partir del Marco Geoestadístico Municipal 2015 (INEGI, 2015a). Del SNIE se recuperaron las coordenadas de cada institución para su geolocalización. Es importante señalar que el SNIE contabilizaba por separado cada sede, escuela o facultad de las IES. Así, por ejemplo, en Ciudad Universitaria de la UNAM, cada facultad es representada como un punto en el mapa. Se tomó como referencia los datos del SNIE 2015-2016 por ser la última versión actualizada disponible. Actualmente el Sistema de Información y Gestión Educativa (SIGED) ofrece los datos de geolocalización de las escuelas; sin embargo, las bases de datos no indican a qué ciclo escolar pertenece la información que reportan. Por su parte, el Marco Geoestadístico permitió la ubicación espacial de las instituciones identificadas en el Sistema.

Para dar cuenta de las condiciones globales de desarrollo de la ciudad, así como del contexto social de la demanda por alcaldía, incluimos los datos del Índice de Desarrollo Humano para México 
de 2015 (PNUD, 2015). El IDH es un indicador que sintetiza los avances logrados en tres dimensiones elementales del desarrollado humano: salud, educación e ingreso. Asimismo, el IDH tiene como objetivo medir el conjunto de capacidades y libertades que tienen los individuos para elegir entre formas de vida alternativas. Para ello, se toman en cuenta tres dimensiones básicas para el desarrollo: 1) la posibilidad de gozar de una vida larga y saludable; 2) la capacidad de adquirir conocimientos; 3) la oportunidad de tener recursos que permitan un nivel de vida digno (PNUD, 2016). Además, estudios previos (Gil Antón et al., 2009) han mostrado la utilidad de usar el IDH como indicador de las condiciones socioeconómicas y de bienestar de una población. Finalmente, la última variable en juego es población joven, que refiere al número de jóvenes de entre 15 y 24 años por municipio o alcaldía, como una aproximación a la población que está en edad de cursar la educación superior o que en algún momento lo puede hacer. Este último cálculo se hizo con base en los datos de la Encuesta Intercensal del INEGI (2015b).

De acuerdo con las delimitaciones mexicanas más utilizadas, la ZMVM abarca alrededor de $7866 \mathrm{~km}^{2}$, comprende las 16 alcaldías de Ciudad de México (antes Distrito Federal), 59 municipios del Estado de México y un municipio de Hidalgo (Tizayuca). La ZMVM tiene más de 20 millones de habitantes, cifra que equivale a $17 \%$ de la población nacional (OECD, 2015).

\section{ANÁLISIS}

\section{La pertinencia socioespacial de los IT}

En esta primera parte del análisis buscamos arrojar luz sobre la pertinencia socioespacial de los IT. Entendemos por pertinencia socioespacial la oportuna ubicación de la oferta de ES con respecto de la demanda potencial y de espacios con diferentes rezagos sociales, en este caso el nivel de desarrollo humano en la Ciudad de México. 


\section{Ubicación}

Una característica notoria de la oferta de ES en la Ciudad de México es su aglomeración en las alcaldías centrales de la capital mexicana y su disminución y alta dispersión en las periferias (Terrazas y Rodríguez, 2017, Taborga, 2002). Esta concentración parece ser una tendencia más clara entre las universidades privadas que entre las públicas. De acuerdo con un estudio, las IES privadas creadas en la ciudad entre 1995 y 2005 tendieron a establecerse en zonas centrales con acceso a amplias vías de comunicación y transporte masivo, como las avenidas Insurgentes y Tlalpan. De aquí que es posible observar que las IES privadas en las alcaldías centrales se articulan primordialmente de norte a sur, siguiendo el trazo de estas avenidas (Uribe, 2011). En cambio, la oferta pública se caracteriza por concentrarse en dos polos: el sur, con la Ciudad Universitaria de la UNAM como principal referente, y el norte, donde destacan la unidad Zacatenco del IPN y la de Azcapotzalco de la UAM.

En el caso de los IT federales, éstos se localizan, predominantemente, en la franja oriente de la Ciudad de México, en paralelo a la frontera entre la ciudad y el Estado de México (mapa 1). También es posible observar que los IT son más numerosos en la zona sur de esta franja que en el norte. En el mapa 1 se incluye el número de IES por cada alcaldía o municipio de la ZMVM. De esta manera, observamos la contribución positiva de los IT asentados en las alcaldías Tláhuac y Milpa Alta ante la falta de oferta de ES en esas localidades. Por el contrario, tres de los doce tecnológicos federales se ubican en alcaldías con alta concentración de escuelas de ES (de 34 a 51 escuelas), a saber, los dos institutos en Gustavo A. Madero y el instituto de Álvaro Obregón. El resto de los IT se ubican en alcaldías con una oferta intermedia, de por lo menos tres planteles. Un último detalle que destacar es la poca cercanía entre los IT federales y descentralizados, siendo las sedes de Iztapalapa II y III y el TES La Paz, en la zona oriente, las de mayor proximidad. 
Mapa 1. Localización de Tecnológicos descentralizados y federales en la Zona Metropolitana del Valle de México en relación con la oferta de ES

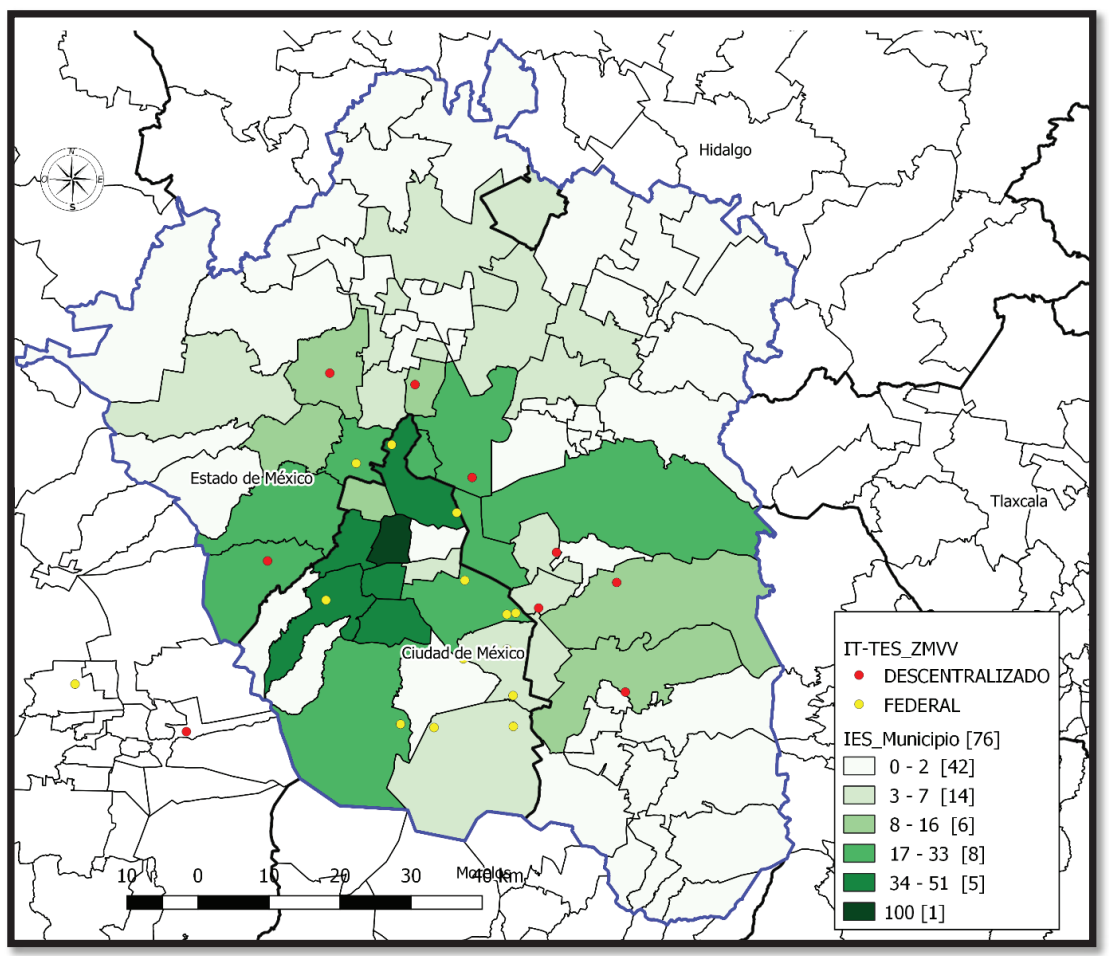

Fuente: elaboración propia a partir de la base de datos SNIE 2015-2016 (SEP).

\section{Nivel de desarrollo}

Para acercarnos al contexto socioeconómico de la demanda potencial de ES de los IT, utilizamos el IDH para cada alcaldía o municipio. Como contexto general, México cuenta con un desarrollo humano alto (0.762), y ocupa la posición 77 a nivel mundial de este indicador. En términos poblacionales, una minoría de los mexicanos (1.23\%) vive en municipios con IDH bajo; otro $19.73 \%$ habita en demarcaciones con un IDH medio, mientras que $41.56 \%$ y $37.49 \%$ reside en municipios con IDH alto o muy alto, respectivamente (PNUD, 2019). En el mapa 2 presentamos el IDH en la ZMVM por municipio o alcaldía. Podemos observar que los municipios y alcaldías que conforman la región poseen IDH medios, altos y muy altos, con los índices más altos concentrados en las alcaldías centrales de la Ciudad de México 
(tono azul más oscuro). Por el contrario, las alcaldías Tláhuac y Milpa Alta, al sur de la capital, cuentan con los niveles de desarrollo más bajos de la ciudad, colindando al mismo tiempo con algunos de los municipios del Estado de México con menor desarrollo.

- Mapa 2. Ubicación de los IT y de TES en la Zona Metropolitana del Valle de México en relación con el Índice de Desarrollo Humano

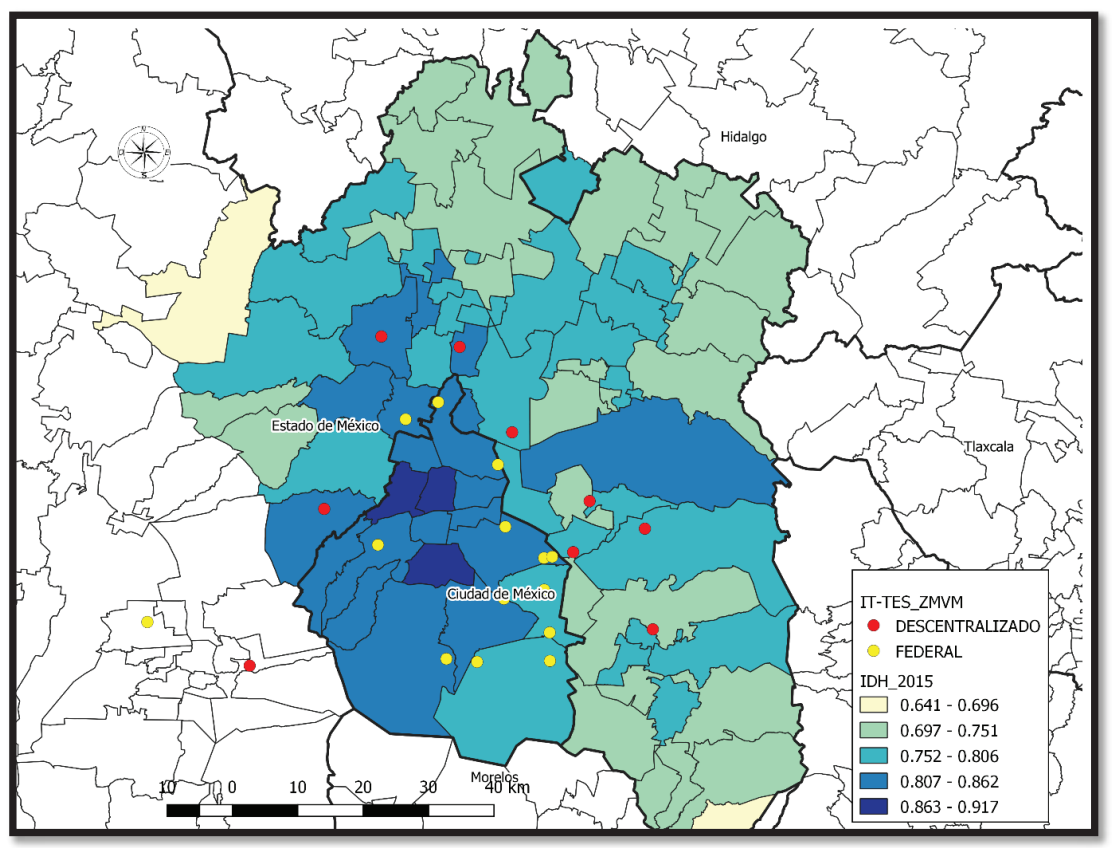

Fuente: elaboración propia a partir de la base de datos SNIE 2015-2016 (SEP) y IDH por municipio (PNUD, 2015).

$\mathrm{Al}$ enforcarnos en la ubicación de los IT, el mapa 2 nos permite apreciar que la misión con la que surgieron, de atender la demanda juvenil más vulnerable, se cumple en la ciudad, aunque parcialmente a nivel metropolitano. Por una parte, todas las sedes de este subsistema educativo se ubican en las alcaldías de menor desarrollo relativo de la ciudad. No obstante, es necesario confirmar el origen geográfico de cada IT para saber si se cumple con la pertinencia geográfica. Por otra parte, al considerar a los IT descentralizados (puntos rojos) en el análisis, podemos observar que aún hay espacios con menor desarrollo humano que no cuentan con oferta de este subsistema, como las regiones norte y sur del Estado de México. 


\section{Población joven}

La última capa que utilizamos en este primer acercamiento al contexto espacial de los IT es el relativo a la población joven (15 a 24 años) de cada municipio y alcaldía de la región. En el mapa 3 observamos que los municipios con mayor cantidad de jóvenes son Iztapalapa en la Ciudad de México y Ecatepec de Morelos en el Estado de México, seguidos por Nezahualcóyotl y Gustavo A. Madero, colindantes a los primeros, y Tlalpan y Álvaro Obregón al sur poniente de la ciudad capital. Destaca el bajo número de jóvenes en la parte poniente y central de la ciudad, las áreas con los niveles de desarrollo más altos (ver mapa 2). Desde esta perspectiva, podemos observar que los IT, tanto federales como descentralizados, se ubican en los municipios con mayor cantidad de jóvenes de la región, sugiriendo que las dinámicas demográficas son un factor importante de la demanda educativa en estos espacios.

Mapa 3. Distribución de las sedes del TecNM en municipios y alcaldías de la ZMVM, en relación con la población joven

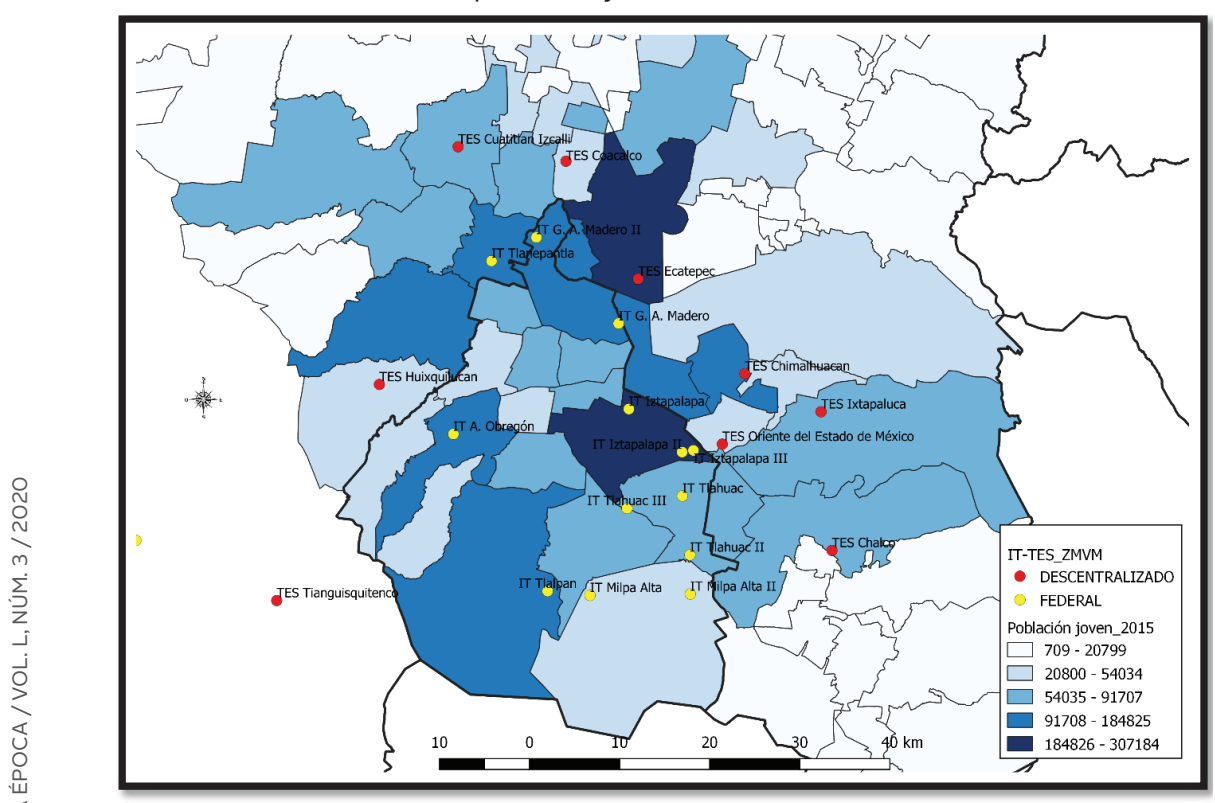

Fuente: elaboración propia a partir de la Encuesta Intercensal del INEGI (2015b). 


\section{Baja demanda de los IT}

En septiembre de 2008 comenzaron a operar los tres primeros institutos tecnológicos en las alcaldías Iztapalapa, Milpa Alta y Tláhuac, en la capital mexicana. A éstos siguieron los institutos de Gustavo A. Madero, dos unidades más en Iztapalapa (Iztapalapa II y III) y otras dos en Tláhuac (Tláhuac II y III). Finalmente, en 2010 comenzaron a operar las unidades Milpa Alta II, Álvaro Obregón, Tlalpan y Gustavo A. Madero II en 2012. El crecimiento en la matrícula de los IT en la CDMX ha sido diferenciado, a pesar de que hay tendencia al alza de la matrícula en cada sede. También podemos observar un crecimiento heterogéneo entre ellas. En este sentido, el IT de Tláhuac es el campus con la mayor matrícula de los IT, superando los más de 2500 estudiantes desde 2013, como se puede apreciar en la gráfica 1.

- Gráfica 1. Crecimiento de la matrícula de los IT en la Ciudad de México, 2008-2018

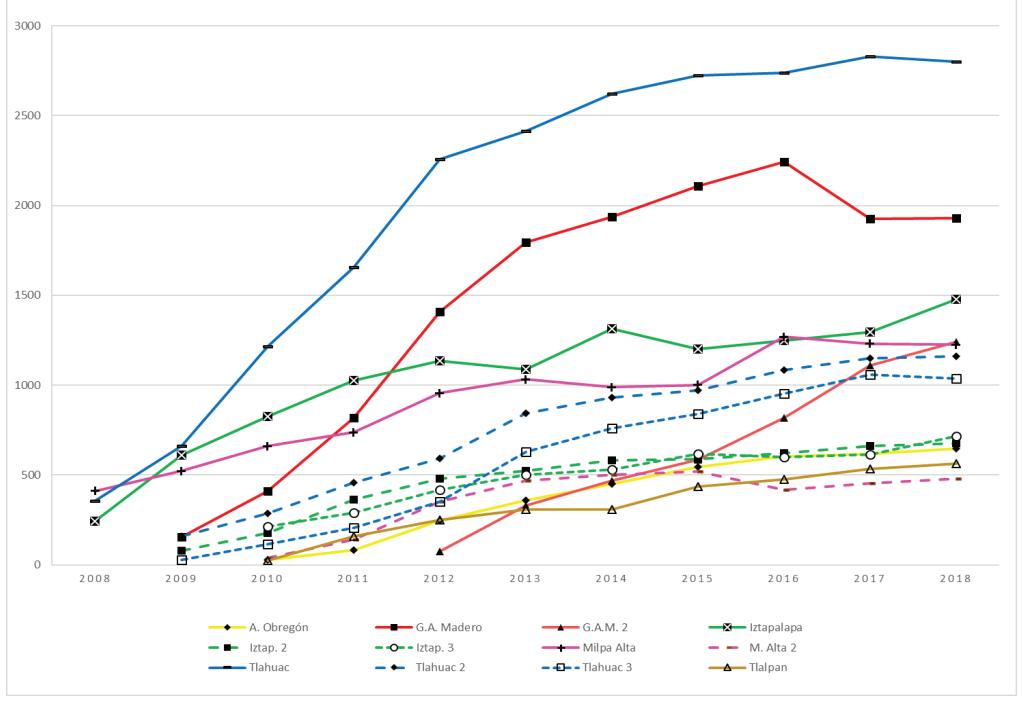

Fuente: anuarios estadísticos de ANUIES (2019).

El tecnológico con la segunda mayor población estudiantil es el IT de Gustavo A. Madero, que ha experimentado una leve disminución en los últimos ańos. Por otra parte, es interesante notar el 
rápido crecimiento de Gustavo A Madero II a tan sólo seis años de su establecimiento, superando ya a la matrícula de Milpa Alta, uno de los tres primeros tecnológicos de la ciudad. En los otros casos, la matrícula promedia los mil estudiantes, asemejándose, en este sentido, a la tendencia observada en las instituciones privadas independientes de absorción de demanda y reciente creación.

Con la finalidad de arrojar un poco de luz sobre este desigual crecimiento analizamos las tres variables usadas en el apartado anterior (oferta de ES, IDH y Población joven), todos por municipio. Se buscaron correlaciones entre estas tres variables y la matrícula total de cada sede; sin embargo, las correlaciones fueron bajas, coeficientes Rho de Spearman de 0.013 oferta de ES; 0.111 Población joven; $-0.288 \mathrm{IDH}$.

Sin embargo, los mapas nos sugieren que tanto el IT Tláhuac como los dos planteles en Gustavo A. Madero colindan con municipios y alcaldías con alta población joven. En el caso de Tláhuac, esta alcaldía se encuentra próxima a Iztapalapa, la de mayor población joven de la ciudad; en el caso de los IT de la GAM, éstos se localizan entre Ecatepec y Nezahualcóyotl, como se puede apreciar en el mapa 3.

Debido a que los municipios de Ecatepec, Nezahualcóyotl y la alcaldía Iztapalapa son las regiones más pobladas del país (en tono más oscuros en el mapa 3), quizá esto genere un "excedente" de población joven que no puede encontrar opciones de ES en su lugar de origen y les orille a ingresar a una opción en otra demarcación, pero que les resulte relativamente cercana. Esta tendencia sugiere que los IT de la GAM y de Tláhuac no sólo ayudan a la absorción de la demanda de la propia demarcación, sino también de las alcaldías o municipios vecinos, en particular de aquellos que tienen poblaciones muy grandes. De igual forma, por la cantidad de jóvenes, se justifica que Iztapalapa sea la alcaldía capitalina con el mayor número de sedes del TecNM.

\section{DISCUSIÓN}

En este trabajo exploramos la pertinencia socioespacial del sistema de institutos tecnológicos federales asentados en la Ciudad de México desde 2008, tomando como eje de análisis la misión con 
la que fueron establecidos en la ciudad: la descentralización de la oferta educativa hacia las alcaldías con menor oferta. Los mapas presentados en los apartados anteriores nos ayudan a confirmar que los IT de la ciudad cumplen con su misión de descentralizar la oferta hacia las entidades locales con menor número de IES, con los contextos de desarrollo más bajos a nivel regional y con un número alto de demanda potencial. En otras palabras, los IT han incrementado las oportunidades educativas de la ciudad. El efecto de "oasis educativo” parece ser más evidente en las alcaldías de Tláhuac y Milpa Alta debido a que éstas se caracterizan por tener los niveles de desarrollo humano más bajos de la ciudad y por contar con una escasa oferta de IES.

A pesar del aparente desajuste entre los propósitos con los que se creó el sistema de educación superior tecnológica (EST) y la distribución de sus sedes en la Ciudad de México, existe un problema que los IT atienden: la demanda potencial. Los IT de la ZMVM tienden a ubicarse en las alcaldías y municipios con más jóvenes. Sin embargo, las diferencias entre el crecimiento de la matrícula de los IT de la Ciudad de México nos sugieren una hipótesis: las sedes con más estudiantes no sólo atienden la demanda local, sino también absorben parte de la demanda de los municipios o alcaldías vecinas. Este fenómeno se da en particular cuando en las localidades vecinas la población joven es muy grande, como en el caso de los IT de la Gustavo A. Madero y los municipios de Ecatepec y Nezahualcóyotl. Este fenómeno también daría cuenta de la movilidad estudiantil interestatal o entre localidades que ocurre en la ZMVM como la identificada por Malo y Hernández (2014, s. p.):

Además de los objetivos planteados al inicio de este trabajo, los mapas nos ayudan a plantear una hipótesis de relevancia para nuestra investigación. Observamos que los doce IT de la Ciudad de México tienden a localizarse de manera esparcida en la franja oriente de la Ciudad de México, mientras que la mayoría de las IES se concentran en las alcaldías centrales. Esto nos sugiere un patrón de segregación espacial de los IT respecto de la oferta tradicional universitaria. Por un lado, esta ubicación particular de los IT podría tener efectos negativos sobre la toma de decisiones de los estu- 
diantes en tránsito hacia la ES pues, de acuerdo con Bayón, Saraví y Ortega (2013), la zona oriente de la ciudad suele ser asociada entre los jóvenes como un espacio de pobreza y peligro. Así, este "estigma territorial" podría inhibir a la potencial demanda proveniente de otras zonas de la ciudad o incluso de la misma área, en el caso de que los jóvenes busquen escapar de estas asociaciones (Bayón, et al., 2013).

Aunado a esto, es importante señalar que la oferta de programas educativos que ofrecen los doces planteles del TecNM en la ciudad se limita a carreras tecnológicas. En la Ciudad de México, la demanda de ES suele concentrarse, primordialmente, en cuatro campos de estudio: derecho, contaduría, administración y medicina (Taborga, 2002). Así, la oferta educativa del TecNM no responde, de entrada, a las preferencias más populares entre los jóvenes, quienes buscarán otras alternativas en distintos espacios de la ciudad.

Por último, es necesario aclarar que este sólo es un primer acercamiento a lo que ofrecen las sedes del TecNM en la Ciudad de México y ZMVM. Queda pendiente por analizar otras variables como el radio de alcance de cada escuela; el peso de la cercanía del campus y las vías de comunicación en la elección de los jóvenes; las tasas de retención, terminación de estudios y titulación, así como el posible aporte de la EST en los procesos de movilidad social de sus egresados.

\section{COMENTARIOS FINALES}

En un país como México, donde sólo tres de cada diez jóvenes en edad universitaria pueden acceder a la ES, la apertura de una nueva IES pública implicará, en todo momento, una ampliación de oportunidades. Sin embargo, en la mayoría de los casos se trata de oportunidades adueñadas por jóvenes de estratos socioeconómicos medios. En este trabajo intentamos aproximarnos a la ampliación de oportunidades educativas en la ZMVM a partir de la apertura de los doce planteles del TecNM en la Ciudad de México. Confirmamos, por un lado, la pertinencia socioespacial de los doce IT, al tiempo que presentamos varias lecturas sobre la baja demanda y el crecimiento desigual de cada plantel. 
A lo largo de este trabajo abogamos por una mayor recepción de la geografía de la educación en la investigación educativa mexicana. Como hemos hecho notar, las herramientas generadas para el análisis de los datos han sido de gran utilidad para observar algunos patrones significativos de la distribución espacial de las IES en la principal región metropolitana de México. Además, hacemos un llamado a atender la dimensión geográfica de las oportunidades educativas en el contexto mexicano y a generar mayor evidencia empírica. Presentamos este artículo como una aproximación en este sentido.

Por otra parte, buscamos promover un mayor impacto de la perspectiva geográfica en la política pública educativa. Por ejemplo, en Reino Unido, las políticas educativas de inclusión social (widening participation policies) buscan dar cuenta de la dimensión geográfica en la desigualdad educativa mediante indicadores como el low-participation neighborhood (LPN) o barrio de baja participación, el cual busca identificar los barrios del país donde el rezago en educación universitario es históricamente persistente. Una vez identificados, estos espacios son objeto de programas de vinculación y reclutamiento por parte de las universidades más cercanas (Harrison y McCaig, 2014). En México, una acción similar podría ser una excelente alternativa a las políticas actuales.

\section{REFERENCIAS}

Asociación Nacional de Universidades e Instituciones de Educación Superior (ANUIES) (2019). Anuario educación superior-licenciatura (2018-2019). México: ANUIES. Recuperado de http://www.anuies. $\mathrm{mx} /$ informacion-y-servicios/informacion-estadistica-de-educacionsuperior/anuario-estadistico-de-educacion-superior

Bayón, M. C., Saraví, G., y Ortega, M. (2013). The Cultural Dimensions of Urban Fragmentation: Segregation, Sociability, and Inequality in Mexico City. Latin American Perspectives, 40(2), 35-52.

Consejo Nacional para la Cultura y las Artes (Conaculta) (2012). Atlas de infraestructura cultural de México. México: Conaculta.

De Garay, A. (2003). El perfil de los estudiantes de nuevo ingreso de las Universidades Tecnológicas en México. El Cotidiano, 19(122), 75-85. 
Diario Oficial de la Federación (2014). Decreto que crea el Tecnológico Nacional de México. México: Gobierno de México. Recuperado de http://www.dof.gob.mx/nota_detalle.php?codigo=5353459\&fec $\mathrm{ha}=23 / 07 / 2014$

Didou, S. (1997). Descentralización y urbanización del Sistema de educación superior: un estado del arte y algunos hechos concretos. Revista Mexicana de Investigación Educativa, 2(3), 31-44.

Didou, S. (2011). La promoción de la equidad en la educación superior en México: declinaciones múltiples. Reencuentro, UAM Xochimilco, (61), 7-18.

Dirección General de Educación Superior Tecnológica (DGEST) (2009). Se crearán 10 institutos tecnológicos en el Distrito Federal durante 2009. Conferencia de Prensa. México: DGEST / DDC. Recuperado de http://www.dgest.gob.mx/dgest/se-crearan-10-institutos-tecnologicos-en-el-distrito-federal-durante-2009

Gil Antón, M.. Mendoza, J., Rodríguez, R., y Pérez, M. J. (2009). Cobertura de la Educación Superior en México. Tendencias, retos y perspectivas. México: ANUIES.

Harrison, N., y McCaig, C. (2014). An ecological fallacy in higher education policy: the use, overuse and misuse of low participation neighborhoods. Journal of Further and Higher Education, (39), 739-817.

Hillman, N. (2016). Geography of College Opportunity: The Case of Education Deserts. American Educational Research Journal, (53), 9871021.

Hillman, N. (2017). Geospatial Analysis in Higher Education Research. En M. B. Paulsen (ed.), Higher Education: Handbook of Theory and Research, vol. 32 (pp. 529-575. Iowa: Cham, Springer. https://doi. org/10.1007/978-3-319-48983-4_11

Hillman, N., y Weichman, T. (2016). Education Deserts, The Continued Significance of "Place" in the Twenty-First Century. Washington DC: American Council on Education, Viewpoints: Voices from the Field.

Instituto Nacional de Estadística y Geografía (INEGI) (2015a). Marco Geoestadístico Nacional. México: INEGI. Recuperado de http://www. inegi.org.mx/geo/contenidos/geoestadistica/m_geoestadistico.aspx

Instituto Nacional de Estadística y Geografía (INEGI) (2015b). Encuesta Intercensal 2015. Recuperado de https://www.inegi.org.mx/programas/intercensal/2015/ 
Lopez Turley, R. (2009). College Proximity: Mapping Access to Opportunity. Sociology of Education, 82(2), 126-146.

Malo, S., y Hernández, A. (2014). Oferta y demanda de educación superior. Este País. Recuperado de http://archivo.estepais.com/site/2014/ oferta-y-demanda-de-educacion-superior/

Mejía, G. (2019). Desconcentración de la educación superior pública, movilidad social y espacial. Un estudio de caso desde la geografía de la educación. (Tesis de doctorado). Departamento de Investigaciones Educativas, Centro de Investigación y de Estudios Avanzados del Instituto Politécnico Nacional, México.

Mejía, G., y Worthman, S. (2015). La descentralización de la educación superior en México a través de las universidades autónomas: un análisis preliminar de los contextos y condiciones sociales. XIII Congreso Nacional de Investigación Educativa. Chihuahua. Comie, México.

Mejía, G., y Worthman, S. (2017). La geografía de las oportunidades. El caso de las sedes de las universidades autónomas en municipios con poca oferta de educación superior. Revista Iberoamericana de Educación Superior, VIII(23), 25-48. https://doi.org/10.22201/ iisue.20072872e.2017.23.3009

Mier, M., y Rabell, C. (2003). Families' and children's inequalities. Journal of Comparative Family Studies, 34(3), 435-454.

Organización para la Cooperación y el Desarrollo Económicos (OCDE) (2015). Valle de México. Sintesis del Estudio. Estudios Territoriales. París: OCDE. http://dx.doi.org/10.1787/9789264245174-en

Olaya, V. (2014). Sistemas de información geográfica. México: Autor. Recuperado de http://volaya.github.io/libro-sig/

Ortega, J. C., y Casillas, M. A. (2013). Nueva tendencia en la educación superior, la oferta en zonas no metropolitanas. Revista de la Educación Superior, 42(167), 63-95.

Programa de las Naciones Unidas para el Desarrollo (PNUD) (2015). Indice de Desarrollo Humano municipal en México: nueva metodología. México: PNUD.

Programa de las Naciones Unidas para el Desarrollo (PNUD) (2016). Informe sobre desarrollo humano. México, 2016. México: PNUD.

Programa de las Naciones Unidas para el Desarrollo (PNUD) (2019). Transformando México desde lo local. Informe de Desarrollo Municipal 2010-2015. México: PNUD. 
Rodríguez, R. (1998). Expansión del sistema educativo superior en México 1970-1995. En M. Fresán (ed.), Tres décadas de políticas del Estado en la educación superior (pp.167-205). México: ANUIES.

Ruiz-Larraguivel, E. (2011). La educación superior tecnológica en México. Historia, situación actual y perspectivas. Revista Iberoamericana de Educación Superior, II(3), 35-52.

Secretaría de Educación Pública (SEP) (2016). Sistema Nacional de Información y Gestión Educativa. México: SEP. Recuperado de https:// www.siged.sep.gob.mx/SIGED/escuelas.htmlhttp

Taborga, H. (2002). Oferta y demanda de estudios de licenciatura en la Zona Metropolitana de la Ciudad de México. México: ANUIES.

Taborga, H. (2003). Expansión y diversificación de la matrícula de la educación superior en México. México: ANUIES.

Terrazas, O., y Rodríguez, M. A. (Coords.) (2017). La universidad accesible. México: Universidad Autónoma Metropolitana-Azcapotzalco.

Uribe, H. (2011). Crecimiento de la educación superior privada y su patrón de distribución territorial en la zona metropolitana de la ciudad de México en 1995 y 2005. (Tesis de maestría). Universidad Autónoma Metropolitana, México.

Weiss, E., y Bernal, E. (2013). Un diálogo con la historia de la educación técnica en México. Perfiles Educativos, 35(139), 151-170. 